\title{
Avaliação da relação velocidade-precisão em função do nível de dificuldade no transtorno de Asperger
}

\author{
Speed-precision assessment in difficulty \\ level function in Asperger's disorder
}

Deise Mara Mota Silva1 ${ }^{1}$, Denise Cardoso Ribeiro², Gilda Pena de Rezende ${ }^{3}$, Talita Dias da Silva ${ }^{4,5}$, Carlos Bandeira de Mello Monteiro², Isabela Lopes Trevizan², Alessandra Bernardes Caturani Wajnsztejn ${ }^{6}$, Thais Massetti²

1Universidade São Caetano do Sul (USCS) - São Caetano do Sul (SP), Brasil.

2Programa de Pós-graduação em Ciências da Reabilitação, Faculdade de Medicina, Universidade de São Paulo (FMUSP) - São Paulo (SP), Brasil.

${ }^{3}$ Grupo de Atendimento Psicopedagógico Integrado (Gapi), Escola Especial - São Bernardo do Campo (SP), Brasil.

${ }^{4}$ Universidade Federal de São Paulo (UNIFESP) - São Paulo (SP), Brasil.

${ }^{5}$ Harvard School of Public Health (HSPH) - Boston (MA), EUA

${ }_{6}^{6}$ Faculdade de Medicina do ABC (FMABC) - Santo André (SP), Brasil.

\section{RESUMO}

Introdução: O Transtorno de Asperger (TA) é considerado um distúrbio do neurodesenvolvimento, caracterizado por deficiências sociais, dificuldade de comunicação, padrões restritos e repetitivos de comportamento. Objetivo: Comparar o desempenho motor na realização de tarefa computacional com base na relação velocidade e precisão, entre pessoas com desenvolvimento típico e com TA, por meio do tempo de movimento. Métodos: Participaram deste estudo oito meninos com diagnóstico de TA e oito meninos com desenvolvimento típico, pareados por idade e sexo, para compor o grupo controle. A tarefa que utilizamos avalia a relação velocidade e precisão de movimento, que consiste na realização de movimentos manuais direcionados a um alvo, em três índices de dificuldade (ID). O tempo de movimento foi obtido por meio da divisão entre segundos pré-estabelecidos para a tarefa (10) e o número de toques realizados no alvo. A ANOVA para medidas repetidas foi utilizada para a comparação entre os ID e entre os grupos. Resultados: Observamos que o tempo de movimento aumentou conforme o progresso dos ID. Conclusão: Os indivíduos com TA apresentaram um tempo de movimento significativamente maior quando comparados ao grupo controle, em todos os ID. Supõe-se que há dificuldades inerentes à doença, e condições motoras dos indivíduos com TA limitam o desempenho durante a tarefa.

Palavras-chave: medição de velocidade; desempenho psicomotor; síndrome de Asperger; estudos de tempo e movimento; lateralidade funcional; software.

\begin{abstract}
Introduction: Asperger's Disorder (AD) is one of the neurodevelopmental disorders characterized by social impairments, difficulty in communication and restricted and repetitive patterns of behavior. Objective: To compare the motor performance in performing computational tasks with base on the relation speed and accuracy among people with typical development and AD, through the time of movement. Methods: The study included eight boys diagnosed with $A D$ and eight children with typical development, matched for age and sex, to compose the control group. The task used to assesses the relative movement speed and accuracy, consisting in making hand movements directed at a target in three levels of difficulty (DI). Movement time was obtained by dividing the seconds to preestablished between the task (10) and the number of rings made on the target. ANOVA for repeated measures was used to compare the DI and between groups. Results: We observed that the movement time increased with the progress of DI. Conclusion: Individuals with $A D$ had a significantly increased movement time compared to the control group in all DI. It is assumed that the difficulties are inherent in motor pathology and conditions of the patients with MT limit performance during task.
\end{abstract}

Keywords: velocity measurement; psychomotor performance; Asperger syndrome; time and motion studies; functional laterality; software. 


\section{INTRODUÇÃO}

O transtorno de Asperger (TA) é definido como alterações do neurodesenvolvimento, caracterizado por deficiências sociais, dificuldade de comunicação e padrões restritos e repetitivos de comportamento $^{1-3}$. O TA apresenta uma prevalência de aproximadamente 2/100 mil pessoas ${ }^{4}$, considerada uma doença rara e que ocorre principalmente em pessoas do sexo masculino ${ }^{4,5}$. A patogênese não é completamente conhecida, mas provavelmente está relacionada à etiologia genética com taxas altas de recorrência familiar ${ }^{6}$.

Os trabalhos em TA apresentam dados referentes aos prejuízos na interação social e no interesse interpessoal ${ }^{7}$, comportamento social limitado ${ }^{8}$, alterações na coordenação ${ }^{7}$, comprometimento cerebelar ${ }^{9}$, neuromotor ${ }^{3}$ e nas habilidades cognitivas ${ }^{10}$. No entanto, não foram encontrados trabalhos direcionados para o controle motor de movimentos em pessoas com TA.

Apesar das disfunções motoras serem classificadas como "sintomas associados", alguns estudos relatam a existência de déficits de coordenação bastante evidentes. Rinehart et al. ${ }^{11}$ analisaram a cinemática do movimento corporal em indivíduos com autismo e TA e os resultados sugerem comprometimento motor com falta de destreza motora, sendo que os autores enfatizam a importância de futuras pesquisas para mapear a trajetória do desenvolvimento de anormalidades motoras nesses pacientes.

Johnson et al. ${ }^{12}$, considerando os conhecimentos em comportamento motor, avaliaram 13 indivíduos com TA e autismo e compararam com 12 indivíduos com desenvolvimento típico, por meio de tarefa com feedback visual. Os resultados sugerem comprometimento funcional da rede cerebelar, o que pode dificultar a funcionalidade e informações de feedback visual para melhora de desempenho. Ming et al. ${ }^{2}$ avaliaram 154 pessoas com TA e observaram que o sintoma motor mais comum foi a hipotonia, sendo apresentada em $51 \%$ dos participantes. Fator interessante do estudo foi que a hipotonia e apraxia motora tiveram uma correlação com a idade, ou seja, observou-se prevalência maior entre as crianças mais jovens, sugerindo que déficits de controle motor fino possam ser melhorados com o tempo.

Apesar dos estudos considerando a funcionalidade, pouco se pesquisou sobre o controle motor no TA, principalmente relacionando dois fatores interessantes para o movimento: a velocidade e a precisão. Beamish et al. ${ }^{13,14}$ descrevem uma propriedade intrínseca do controle motor humano, a relação existente entre velocidade e precisão de movimento ${ }^{15}$. A incapacidade de executar ações com rapidez e precisão, simultaneamente, dificulta a realização de tarefas motoras, afetando diretamente o desempenho em tarefas do dia a dia. Existe uma relação inversamente proporcional entre a velocidade com que um segmento corporal é deslocado e a precisão que se obtém com esse movimento, ou seja, o tempo de movimento (TM) que um indivíduo necessita para atingir alvos distantes e/ou estreitos são maiores se comparados ao TM para alvos mais próximos e mais largos, portanto, verifica-se que o TM varia em função da relação entre a distância e largura dos alvos ${ }^{15-18}$.
Em função das possíveis dificuldades motoras apresentadas por pessoas com TA, este estudo tem como objetivo comparar o desempenho motor na realização de tarefa computacional com base na relação velocidade e precisão, proposta por Fitts ${ }^{15}$, entre pessoas com desenvolvimento típico e com TA, por meio do TM, a fim de quantificar essas limitações. Burgstahler et al. ${ }^{19}$ citam que computadores e outros dispositivos de tecnologia assistiva têm potencial para maximizar a independência, produtividade e participação das pessoas com deficiência em programas acadêmicos, emprego, recreação e outras atividades, tornando assim possível para uma pessoa com limitações utilizar recursos de avanços tecnológicos para executar tarefas diárias com sucesso.

A tarefa de Fitts, por meio das restrições espaciais (manipulando o tamanho e distância do alvo), nos permite verificar se a relação velocidade e precisão se reproduz em pessoas com TA e se há diferença de desempenho quando comparadas a pessoas com desenvolvimento típico. Nesse contexto, a relação velocidade e precisão pode subsidiar respostas sobre controle motor em tarefas pré-estabelecidas, o que auxiliará na compreensão da organização do movimento de pessoas com TA, assim como esse conhecimento pode contribuir para um melhor direcionamento de condutas e otimizar intervenções motoras.

\section{MÉTODOS}

\section{Participantes}

Participaram deste estudo 16 pessoas do sexo masculino sendo, 8 com diagnóstico de TA (grupo com Asperger - GA), com média de idade de 10 $\pm 1,5$ anos, que frequentam o Grupo de Atendimento Psicopedagógico Integrado (GAPI) Educação Especial, instituição localizada na cidade de São Bernardo do Campo, São Paulo, especializada em educação para autistas. Foram também avaliadas 8 pessoas com desenvolvimento típico (grupo com desenvolvimento típico - GDT), com idade média de $10 \pm 2,5$ anos, pareados por sexo e idade com o GA, para compor o grupo controle.

Os critérios de inclusão foram: assinatura do Termo de Consentimento Livre e Esclarecido pelos pais ou responsáveis pelos menores, diagnóstico de TA realizado por neurologista infantil e equipe multidisciplinar da instituição por meio de histórico do paciente, avaliação psicológica, avaliação de comunicação e avaliação psiquiátrica. Para inclusão também foi necessária a compreensão da tarefa, avaliada pela capacidade em realizar adequadamente três tentativas explicativas com demonstração pelo avaliador. Os critérios de exclusão foram doenças associadas, como déficit de atenção e hiperatividade (TDAH) e comorbidades, como desordens cognitivas que impossibilitassem a compreensão da tarefa.

\section{Instrumento de coleta de dados e procedimento}

Para avaliar a relação velocidade e precisão, utilizou-se o software Fitts Reciprocal Aiming Task v.1.0 (Horizontal), de domínio público ${ }^{20}$, o qual apresenta a tarefa que relaciona velocidade $\mathrm{e}$ 
precisão em um computador. Utilizamos um notebook da marca ASUS - Ultrabook, com monitor de 14 polegadas, com o software citado instalado, para a realização da tarefa.

As pessoas foram posicionadas em uma mesa, de altura regulável, com o computador instalado à frente. A cadeira foi ajustada de acordo com o tamanho e a necessidade individual, assim como o apoio para os pés, de tal forma que o posicionamento fosse adequado para viabilizar o início e a finalização da tarefa.

Antes de iniciar a tarefa, os procedimentos foram informados verbalmente para cada participante, a pessoa colocou a mão dominante sobre o mouse, sendo que o cursor (visualizado no computador) deveria estar em um ponto intermediário entre os dois alvos (barras laterais). Após a posição inicial, a pessoa foi instruída a utilizar o mouse para movimentar o cursor e clicar em cima dos alvos — duas barras, que ficam dispostas paralelamente na vertical, com dimensões e distância determinadas de acordo com cada índice de dificuldade (ID) - , de forma alternada e mais rápida possível. A pessoa foi informada que poderia iniciar o movimento a partir do alarme sonoro deflagrado pelo computador para início da tarefa (Figura 1).

Após 10 segundos um novo alarme sonoro indicava o fim da tarefa. Em seguida foi registrado o TM total, obtido por meio da divisão entre segundos pré-estabelecidos para a tarefa (10) e o número de toques (10/número de toques). Caso o participante excedesse dois toques errados, deveria repetir a respectiva tarefa.

A tarefa foi composta por três tentativas em cada um dos três índices de dificuldade (ID2, ID4 e ID6), sendo que cada índice teve modificação da largura e distância das barras com crescente nível de dificuldade. O ID4 foi aplicado de duas formas (ID4a e ID4b): no ID4a a distância e a largura das barras são maiores, no ID4b a distância entre as barras e a largura são menores, mantendo o mesmo ID. Dessa forma, os ID e seus parâmetros são: ID2 (barras laterais com 5,08 cm; distância entre barras de 10,16 cm); ID4a (barras laterais com 2,54 cm; distância entre barras de 20,32 cm); ID4b (barras laterais com 1,27 cm; distância entre barras de 10,16 cm); e ID6 (barras laterais com 0,635 cm; distância entre barras de 20,32 cm).

\section{Análise dos dados}

Para análise dos dados foi realizada a média das três tentativas em cada índice de dificuldade (ID2, ID4a, ID4b e ID6). As variáveis dependentes foram submetidas à ANOVA com fator 2 (grupos GA e GDT) por 2 (ID), com medidas repetidas no último fator. O post-hoc de Tukey-HSD foi escolhido para identificar as diferenças, caso houvessem $(\alpha=0,05)$.

\section{RESULTADOS}

\section{Índices de dificuldade ID2-ID4a}

Houve efeito entre os ID $\left[F(1,14)=39,5 ; \mathrm{p}<0,001 ; \mathrm{n}^{2}=0,74\right]$, sem interação entre ID e grupos. Isso significa que ambos os grupos mostraram um pior desempenho no ID4a ( $\mathrm{M}=1,17 \mathrm{~ms})$ quando comparado ao ID2 ( $\mathrm{M=0,79} \mathrm{ms})$. Um efeito entre grupos também foi encontrado no ID $\left[F(1,14)=12,3 ; p=0,003 ; n^{2}=0,47\right]$ (Figura 2), ou seja o GA teve maior TM $(\mathrm{M}=1,20 \mathrm{~ms})$ do que o $\mathrm{GDT}(\mathrm{M}=0,77 \mathrm{~ms})$.

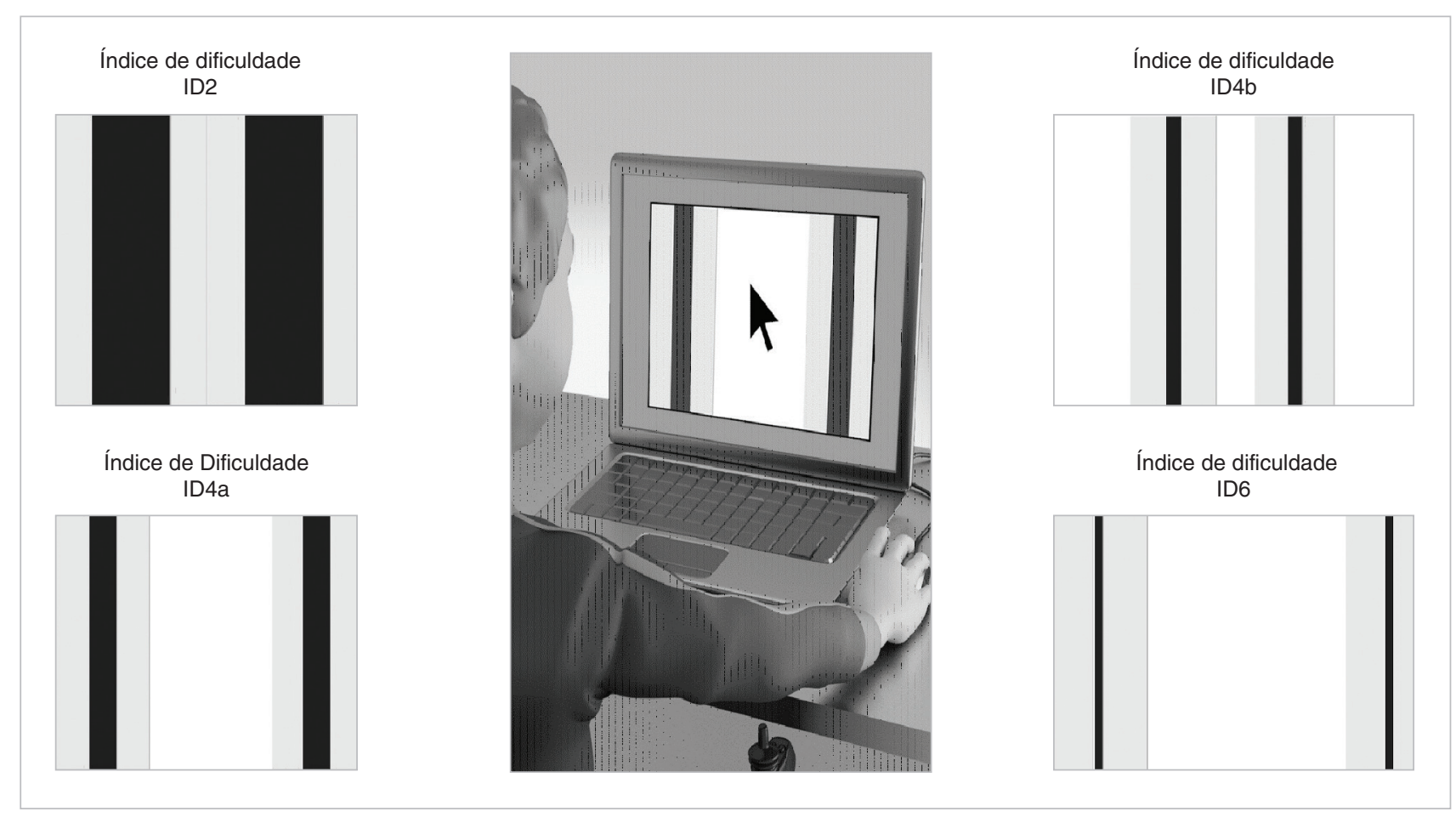

Figura 1: Representação da tarefa de Fitts 


\section{Índices de dificuldade ID4a-ID4b}

Não houve efeitos para ID ou grupos. Interações entre as variáveis também não foram encontradas. Entretanto, houve efeito para grupos $\left[\mathrm{F}(1,14)=11,9 ; \mathrm{p}=0,004 ; \mathrm{n}^{2}=0,46\right]$ (Figura 2). Nos ID4a e ID4b o GA apresentou maior TM $(\mathrm{M}=1,38 \mathrm{~ms})$ quando comparado ao GDT ( $\mathrm{M}=0,91 \mathrm{~ms})$.

\section{Índices de dificuldade ID4b-ID6}

Efeito foi encontrado para os ID $\left[\mathrm{F}(1,14)=23,8 ; \mathrm{p}<0,001 ; \mathrm{n}^{2}=0,63\right]$, entretanto, não houve interação entre ID e grupos. No ID6 o TM foi

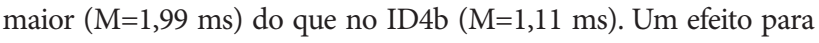
os grupos também foi encontrado $\left[\mathrm{F}(1,14)=7,11 ; \mathrm{p}=0,018 ; \mathrm{n}^{2}=0,34\right]$ (Figura 2), no qual, assim como nos outros ID, o GA apresentou maior TM ( $\mathrm{M}=1,93 \mathrm{~ms})$ quando comparado ao GDT ( $\mathrm{M}=1,17 \mathrm{~ms})$.

\section{DISCUSSÃO}

A TA caracteriza-se por prejuízos na interação social, bem como interesses circunscritos intensos que ocupam totalmente o foco da atenção com comportamentos sociais limitados ${ }^{7,8}$. Verifica-se, também, aquisição atrasada das habilidades motoras, tais como andar de bicicleta, agarrar uma bola, abrir garrafas e subir em brinquedos ao ar livre. Klin ${ }^{7}$ cita que pessoas com TA são visivelmente desajeitadas e têm coordenação limitada.

Embora considerada uma disfunção neurocomportamental, a verificação de alterações no controle motor é interessante para identificar dificuldades funcionais em tarefas do dia a dia. Para tanto, optou-se por avaliar a velocidade e precisão do movimento em uma tarefa no computador e verificar se as pessoas com TA apresentam algum padrão diferente de pessoas com desenvolvimento típico. Huys et al. ${ }^{21}$ citam que o TM pode variar de indivíduo para indivíduo, em função do nível de controle e aquisição

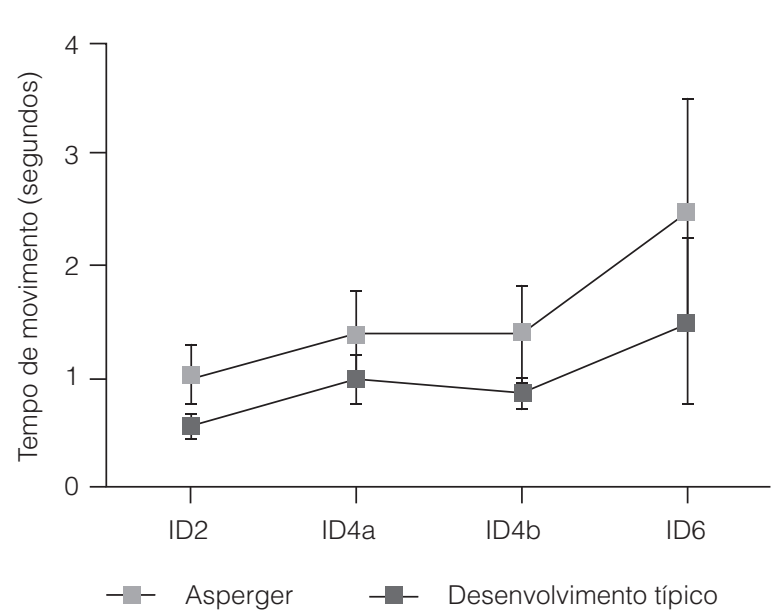

ID: índice de dificuldade

Figura 2: Média do tempo de movimento em cada índice de dificuldade nos dois grupos avaliados motora individual, o que mostra a capacidade de adaptação do sistema nervoso a diferentes tarefas.

Analisando os resultados obtidos, verifica-se que o comportamento apresentado pelos grupos GA e GDT respeitou a relação entre velocidade e precisão de movimento. Os dois grupos apresentaram maior TM à medida que se exige mais precisão, ou seja, conforme ocorre a diminuição da largura ou o aumento da distância entre os alvos, a tarefa se torna mais difícil e obrigatoriamente se observa uma diminuição na velocidade de execução, sendo que essa relação foi observada em ambos os grupos. Beamish et al. ${ }^{13,14}$ citam que o corpo humano tem capacidade limitada para transmitir informações na organização do comportamento motor, sendo assim, o tempo necessário para executar uma tarefa é proporcional à quantidade de informações necessárias para produzir o movimento ${ }^{22}$.

Fitts ${ }^{15}$ estabeleceu que quando a dificuldade do movimento aumenta, pelo aumento da distância ou da diminuição da largura das barras, mais informações devem ser processadas para gerar um movimento que atinja o alvo. Em virtude da premissa de que a capacidade de processamento de informação por unidade de tempo é limitada no executante, há compensação por intermédio do aumento do TM para lidar com uma combinação difícil de distância e largura das barras. Assim, mais tempo é preciso para executar um movimento difícil, sendo que o TM médio é linearmente proporcional ao ID.

Inclusive, para enfatizar a relação velocidade e precisão, não houve diferença estatística entre o ID4a e ID4b para os dois grupos (GDT e GA). No ID4a a distância entre as barras e a largura é maior, enquanto no ID4b a distância entre as barras e a largura das mesmas é menor, dessa forma, a dificuldade se mantém a mesma (com a variação proporcional do tamanho e distância dos alvos).

Apesar do grupo com TA apresentar a mesma característica considerando a velocidade e precisão, observou-se que os desempenhos em todos os ID foram bastante inferiores em relação ao grupo com desenvolvimento típico. Inclusive no ID6 (que representa a tarefa com maior restrição espacial) houve uma diferença acentuada entre os grupos (Figura 2), demonstrando a dificuldade no desempenho motor das pessoas com TA quando há aumento da precisão do movimento.

Provavelmente o pior desempenho das pessoas com TA esteja associado às características motoras observadas em alguns trabalhos, como "alterações motoras"23, "imperícia motora", "falta de jeito" ${ }^{24}$ e "falta de destreza motora"11. Como consequência, alterações em habilidades manuais de direcionamento que requerem desempenho rápido e preciso, sejam mais difíceis para pessoas com TA.

Uma possível justificativa para a diferença de desempenho em pessoas com TA pode se basear em fatores neurofisiológicos. Embora haja poucas pesquisas nessa área, algumas especulações podem ser realizadas. O cerebelo está envolvido no controle das ações motoras finas e na discriminação de informações sensoriais, e sua ação é frequentemente associada com tarefas motoras que 
exigem velocidade e precisão, podendo, portanto, contribuir para alterar o desempenho motor nessas pessoas ${ }^{25,26}$.

Subsidiando essa afirmativa, Gowen e Miall ${ }^{9}$ questionaram o comprometimento cerebelar em pessoas com TA. Para tanto, realizaram testes de apontamento rápido, giro de mão rápida, equilíbrio, força de preensão e tempo de intervalo. Os resultados demonstraram que pessoas com TA têm diminuição de precisão e ritmo, aumento de instabilidade postural e diminuição da cronometragem de precisão, sugerindo que esses déficits específicos refletem prejuízo na capacidade de integrar informações sensoriais com comandos motores apropriados e consistentes, direcionando para disfunções cerebelares ${ }^{9}$. Apesar da hipótese de alterações cerebelares no TA, estudos ainda devem ser realizados, pois existem controvérsias nos resultados.

Segundo Enticott et al. ${ }^{27}$ e Chloe et al. ${ }^{28}$, estudos de imagem cerebral, neurofisiologia e interação cerebelar realizados em autismo sugerem disfunções tanto dos gânglios da base como em regiões fronto-estriatais do cerebelo, no entanto, é pouco claro se isso também se estende de forma tão consistente no TA.

Foram encontrados trabalhos que utilizaram a relação entre velocidade e precisão em diferentes condições neurológicas. Bravo et al. ${ }^{29}$ avaliaram o movimento do braço em seis pessoas com paralisia cerebral (PC) e compararam com seis pessoas com desenvolvimento típico. No resultado observaram que o TM foi linear e positivamente relacionado com o ID para o grupo com movimento normal, mas não para o grupo com PC. Os autores discutem a possibilidade da espasticidade ter influenciado na limitação do movimento. Gump et al. ${ }^{30}$ também avaliaram a relação velocidade e precisão na PC e observaram altas taxas de erro, que podem estar relacionadas aos problemas oculomotores característicos da leão encefálica. Zimmerli et al. ${ }^{31}$ estudaram indivíduos com acidente vascular encefálico e citam que a relação velocidade e precisão deve ser utilizada na prática clínica com o objetivo de viabilizar adaptações de ID. Assim como Lam et al. ${ }^{17}$, verificaram que pessoas com síndrome de Down também eram mais lentas do que o grupo com desenvolvimento típico e, principalmente, apresentaram tempo de permanência maior em cada alvo.

A proposta desta pesquisa foi analisar a performance de pessoas com TA diante do desafio de executar uma tarefa com velocidade e precisão de movimento. Algumas limitações existentes devem ser apresentadas:

1. não existe validação publicada do software utilizado;

2. não foram realizadas avaliações cognitivas no grupo com TA;

3. não foram verificadas diferenças socioeconômicas, experiências prévias com tarefas no computador e convívio com tecnologia; e 4. o difícil diagnóstico possibilitou a avaliação de um número pequeno de pessoas com TA.

Apesar das limitações existentes, acredita-se que os resultados demonstram a capacidade funcional das pessoas avaliadas e contribuam para a organização de programas de reabilitação motora no TA.

Desta forma, os resultados deste estudo nos sugerem que possa haver algum grau de deficiência no planejamento e no controle do movimento durante uma tarefa com exigência crescente de precisão em pessoas com TA quando comparadas a um grupo com desenvolvimento típico. A incapacidade de realizar ações com rapidez e precisão ao mesmo tempo dificulta a realização de tarefas motoras, afetando diretamente seu desempenho, podendo, assim, dificultar atividades da vida diária desses indivíduos.

Conclui-se que, perante os resultados obtidos, a relação velocidade e precisão de movimento são aplicáveis no TA, visto que o TM aumentou conforme o aumento do ID. No entanto, independente do ID, os indivíduos com TA apresentaram um TM significativamente maior se comparados ao grupo controle. Apesar da necessidade de outras pesquisas com base no conhecimento do controle motor, a utilização da relação velocidade e precisão de movimento pode ser um fator interessante na organização de programas de tratamento do TA.

\section{REFERÊNCIAS}

1. Silva EB, Filipini $R$, Monteiro $C B$, Valenti VE, Carvalho SM, Wajnsztejn $R$, et al. The biopsychosocial processes in autism spectrum disorder. Int Arch Med. 2013;6(1):22. http://dx.doi.org/10.1186/1755-7682-6-22

2. Ming $X$, Brimacombe $M$, Wagner GC. Prevalence of motor impairment in autism spectrum disorders. Brain Dev. 2007;29(9):565-70. http://dx.doi.org/10.1016/j.braindev.2007.03.002

3. Nayate A, Tange BJ, Brasshaw JL, McGinley JL, lanset R, Rinehart NJ. Differentiation of high-functioning autism and Asperger's disorder based on neuromotor behavior. J Autism Dev Disord. 2012;42(5):707-17 http://dx.doi.org/10.1007/s10803-011-1299-5

4. Fombonne E. Epidemiology of pervasive developmental disorders. Pediatr Res. 2009;65(6):591-8. http://dx.doi.org/10.1203/PDR.0b013e31819e7203

5. Orrú SE. Síndrome de Asperger: aspectos científicos e educacionais. Rev Iberoam Educ. 2010:53(7):1-14.
6. Volkmar FR, Pauls D. Autism Lancet. 2003:362(9390):1133-41. http://dx.doi.org/10.1016/S0140-6736(03)14471-6

7. Klin A. Autism and Asperger syndrome: an overview. Rev Bras Psiquiatr. 2006;28(Suppl 1):S3-11.

http://dx.doi.org/10.1590/S1516-44462006000500002

8. Watanabe $\mathrm{K}$, Ikeda $\mathrm{H}$, Miyao M. Learning efficacy of explicit visuomotor sequences in children with attention-deficit/hyperactivity disorder and Asperger syndrome. Exp Brain Res. 2010;203(1):233-9. http://dx.doi.org/10.1007/s00221-010-2217-3

9. Gowen E, Miall RC. Behavioural aspects of cerebellar function in adults with Asperger syndrome. Cerebellum. 2005;4(4):279-89. http://dx.doi.org/10.1080/14734220500355332

10. Wilson CE, Happé F, Wheelwright SJ, Ecker C, Lombardo MV, Johnston $\mathrm{P}$, et al. The neuropsychology of male adults with highfunctioning autism or Asperger syndrome. Autism Res. 2014;7(5): 568-81.

http://dx.doi.org/10.1002/aur.1394 
11. Rinehart NJ, Bellgrove MA, Tonge BJ, Brereton AV, Howells-Rankin D, Bradshaw JL. An examination of movement kinematics in young people with high-functioning autism and asperger's disorder: further evidence for a motor planning deficit. J Autism Dev Disord. 2006;36(6):757-67. http://dx.doi.org/10.1007/s10803-006-0118-x

12. Johnson BP, Rinehart NJ, White O, Millist L, Fielding J. Saccade adaptation in autism and Asperger's disorder. Neuroscience. 2013;243:76-87

http://dx.doi.org/10.1016/j.neuroscience.2013.03.051

13. Beamish D, Bhatti S, Chubbs CS, Mackenzie S, Wu J, Jing Z Estimation of psychomotor delay from the Fitts' law coefficients. Biol Cybern. 2009;101(4):279-96.

http://dx.doi.org/10.1007/s00422-009-0336-3

14. Beamish D, Bhatti S, Mackenzie IS, Wu J. Fifty years later: a neurodynamic explanation of Fitts' law. J R Soc Interface. 2006;3(10):649-54

http://dx.doi.org/10.1098/rsif.2006.0123

15. Fitts PM. The information capacity of the human motor system in controlling the amplitude of movement. J Exp Psychol. 1954;47(6):381-91.

16. Fitts PM, Radford BK. Information capacity of discrete motor responses under different cognitive sets. J Exp Psychol. $1966 ; 71(4): 475-82$.

17. Lam MY, Hodges NJ, VIrji-Babul N, Latash ML. Evidence for slowing as a function of index of difficulty in young adults with Down syndrome. Am J Intellect Dev Disabil. 2009;114(6):411-26. http://dx.doi.org/10.1352/1944-7558-114.6.411

18. Vaughan J, Barany DA, Sali AW, Jax SA, Rosenbaum DA. Extending Fitts' Law to three-dimensional obstacle-avoidance movements: support for the posture-based motion planning model. Exp Brain Res. 2010;207(1-2):133-8. http://dx.doi.org/10.1007/s00221-010-2431-z

19. Burgstahler S, Comden D, Lee SM, Arnold A, Brown K. Computer and cell phone access for individuals with mobility impairments: an overview and case studies. NeuroRehabilitation. 2011;28(3):183-97. http://dx.doi.org/10.3233/NRE-2011-0648

20. Okazaki VHA. Softwares de análise de movimento. Disponível em: http://okazaki.webs.com/softwaresdownloads.htm. Acesso em: 01 set. 2010.

21. Huys R, Fernandez L, Bootsma RJ, Jirsa VK. Fitts' law is not continuous in reciprocal aiming. Proc Biol Sci. 2010;277(1685):1179-84. http://dx.doi.org/10.1098/rspb.2009.1954
22. Pelk K, Dan B. Postural cortical myoclonus during gait in Rett syndrome. Epylepsy Behav. 2009;16(1):188.

http://dx.doi.org/10.1016/j.yebeh.2009.06.024

23. Behere A, Shahani L, Noggle CA, Dean R. Motor functioning in autistic spectrum disorders: a preliminary analysis. J Neuropsychiatry Clin Neurosci. 2012;24(1):87-94.

http://dx.doi.org/10.1176/appi.neuropsych.11050105

24. Enticott PG, Bradshaw JL, lansek R, Tonge BJ, Rinehart NJ. Electrophysiological signs of supplementary-motor-area deficits in high-functioning autism but not Asperger syndrome: an examination of internally cued movement-related potentials. Dev Med Child Neurol. 2009;51(10):787-91

http://dx.doi.org/10.1111/j.1469-8749.2009.03270.x

25. Gao JH, Parsons LM, Bower JM, Xiong J, Li J, Fox PT. Cerebellum implicated in sensory acquisition and discrimination rather than motor control. Science. 1996;272(5261):545-7. http://dx.doi.org/10.1126/science.272.5261.545

26. Winstein CJ, Grafton ST, Pohl PS. Motor task difficulty and brain activity: investigation of goal-directed reciprocal aiming using positron emission tomography. J Neurophysicol. 1997; $77(3): 1581-94$

27. Enticott PG, Rinehart NJ, Tonge BJ, Bradshaw JL, Fitzgerald PB. A preliminary transcranial magnetic stimulation study of cortical inhibition and excitability in high-functioning autism and Asperger disorder. Dev Med Child Neurol. 2010;52(8):e179-83. http://dx.doi.org/10.1111/j.1469-8749.2010.03665.x

28. Stanley-Cary C, Rinehart N, Tonge B, White O, Fielding J. Greater disruption to control of voluntary saccades in autistic disorder than Asperger's disorder: evidence for greater cerebellar involvement in autism? Cerebellum. 2011;10(1):70-80. http://dx.doi.org/10.1007/s12311-010-0229-y

29. Bravo PE, LeGare M, Cook AM, Hussey S. A study of the application of Fitts' law to selected cerebral palsied adults. Percept Mot Skills. 1993;77(3 Pt 2):1107-17. http://dx.doi.org/10.2466/pms.1993.77.3f.1107

30. Gump A, LeGare M, Hunt DL. Application of Fitts' law to individuals with cerebral palsy. Percept Mot Skills. 2002;94(3 Pt 1):883-95 http://dx.doi.org/10.2466/pms.2002.94.3.883

31. Zimmerli L, Krewer C, Gassert R, Müller F, Riener R, Lünenburger L. Validation of a mechanism to balance exercise difficulty in robotassisted upper-extremity rehabilitation after stroke. J Neuroeng Rehabil. 2012;9(6)

http://dx.doi.org/10.1186/1743-0003-9-6. 